Bull. Korean Math. Soc. 46 (2009), No. 6, pp. 1153-1158

DOI 10.4134/BKMS.2009.46.6.1153

\title{
ON SELF-RECIPROCAL POLYNOMIALS AT A POINT ON THE UNIT CIRCLE
}

\author{
SEON-Hong KIM
}

\begin{abstract}
Given two integral self-reciprocal polynomials having the same modulus at a point $z_{0}$ on the unit circle, we show that the minimal polynomial of $z_{0}$ is also self-reciprocal and it divides an explicit integral self-reciprocal polynomial. Moreover, for any two integral self-reciprocal polynomials, we give a sufficient condition for the existence of a point $z_{0}$ on the unit circle such that the two polynomials have the same modulus at $z_{0}$.
\end{abstract}

\section{Introduction and statement of results}

Throughout this paper, $U$ denotes the unit circle and $n$ is a positive integer. A polynomial $P(z)=a_{n} z^{n}+a_{n-1} z^{n-1}+\cdots+a_{0}$ is said to be a self-reciprocal polynomial of degree $n$ if it satisfies $a_{n} \neq 0$ and $P(z)=z^{n} P(1 / z)$. Thus the zeros of a self-reciprocal polynomial either lie on the unit circle or are symmetric with respect to $U$. There have been a number of interesting problems (for example [2]) about the distribution of zeros of self-reciprocal polynomials. Also the minimal polynomial of an algebraic number $\alpha$ is the unique irreducible monic polynomial $f(z)$ of smallest degree with rational coefficients such that $f(\alpha)=0$.

In this paper, we study a generalization of an already rather general problem, that of determining the zeros of a polynomial on $U$. This maybe phrased as finding $z$ with $|z|=1$ such that $|P(z)|=0$, where $P(z)$ is a polynomial. We propose broaden this to the problem for finding $z$ with $|z|=1$ such that $|P(z)|=|Q(z)|$, where $P(z)$ and $Q(z)$ are polynomials. A first priority in this fashion seems to determine the minimal polynomial $F(z)$ of an element of the set

$$
\{z:|P(z)|=|Q(z)|,|z|=1\}
$$

Received September 19, 2008.

2000 Mathematics Subject Classification. Primary 30C15; Secondary 26C10.

Key words and phrases. self-reciprocal polynomials, zeros, unit circle.

This Research was supported by the Sookmyung Women's University Research Grants 2009

(C)2009 The Korean Mathematical Society 
Also, what can be said about the number of zeros on $U$ of $F(z)$ ? We study these questions in case that the polynomials are integral and self-reciprocal.

Now we establish the first result.

Theorem 1. Let $P(z)$ and $Q(z)$ be integral self-reciprocal polynomials with $\operatorname{deg} P(z)=m \geq n=\operatorname{deg} Q(z)$. Suppose that

$$
\left|P\left(z_{0}\right)\right|=\left|Q\left(z_{0}\right)\right| \neq 0
$$

for some $z_{0}$ with $\left|z_{0}\right|=1$ and $z_{0} \neq 1$. Then the minimal polynomial of $z_{0}$ is also self-reciprocal and it divides integral self-reciprocal polynomial $P(z)^{2}-$ $z^{m-n} Q(z)^{2}$.

In above theorem, $z_{0} \neq 1$ is required because the minimal polynomial of 1 is $z-1$ which is not self-reciprocal. One may ask whether there always exist $z_{0}$ with $\left|z_{0}\right|=1$ and $z_{0} \neq 1$ such that $\left|P\left(z_{0}\right)\right|=\left|Q\left(z_{0}\right)\right|$ for any two integral self-reciprocal polynomials $P(z)$ and $Q(z)$. But an example of

$$
P(z)=z^{3}-2 z^{2}-2 z+1, \quad Q(z)=z^{2}-7 z+1
$$

gives the negative answer by Theorem 1 . This is because $P(z)^{2}-z^{m-n} Q(z)^{2}$ has no zeros on $U$. Hence it is interesting to mention the condition that the question above is true. We now give a sufficient condition for that when $P(z)$ and $Q(z)$ are even degrees of polynomials.

Theorem 2. For even integers $m$ and $n$, let

$$
P(z)=\sum_{k=0}^{m} a_{k} z^{k}, \quad Q(z)=\sum_{k=0}^{n} b_{k} z^{k}
$$

be integral self-reciprocal polynomials with $\operatorname{deg} P(z)=m \geq n=\operatorname{deg} Q(z)$. If either

$$
\left(a_{\frac{m}{2}}-b_{\frac{n}{2}}\right)^{2}<\frac{8}{4 m+3}\left[\sum_{k=1}^{\frac{n}{2}}\left(a_{\frac{m}{2}-k}-b_{\frac{n}{2}-k}\right)^{2}+\sum_{k=\frac{n}{2}+1}^{\frac{m}{2}} a_{\frac{m}{2}-k}^{2}\right]
$$

or

$$
\left(a_{\frac{m}{2}}+b_{\frac{n}{2}}\right)^{2}<\frac{8}{4 m+3}\left[\sum_{k=1}^{\frac{n}{2}}\left(a_{\frac{m}{2}-k}+b_{\frac{n}{2}-k}\right)^{2}+\sum_{k=\frac{n}{2}+1}^{\frac{m}{2}} a_{\frac{m}{2}-k}^{2}\right]
$$

then there exists $z_{0} \in \mathbb{C}$ with $\left|z_{0}\right|=1$ such that

$$
\left|P\left(z_{0}\right)\right|=\left|Q\left(z_{0}\right)\right| \text {. }
$$

In Section 2, we provide proofs and some examples of Theorems 1 and 2 . 


\section{Proofs and examples}

Proof of Theorem 1. The first part of the theorem follows from the well known fact that the integral minimal polynomial $f(z)$ of degree $d$ of $z_{0}$ with $\left|z_{0}\right|=1$ is self-reciprocal. This is because

$$
z_{0}^{d} f\left(z_{0}^{-1}\right)=z_{0}^{d} f\left(\overline{z_{0}}\right)=0
$$

and $z_{0}$ is a zero of the polynomial $z^{n} f\left(z^{-1}\right)$ which has degree $d$. Since the minimal is unique, we have $f(z)=z^{d} f\left(z^{-1}\right)$. We now prove the second part of the theorem. Suppose that $P(z)$ and $Q(z)$ are integral self-reciprocal polynomials with $\operatorname{deg} P(z)=m \geq n=\operatorname{deg} Q(z)$. Consider $2 m$ degree polynomial

$$
F(z)=P(z)^{2}-z^{m-n} Q(z)^{2} .
$$

Then $F(z)$ is an integral self-reciprocal polynomial since

$$
\begin{aligned}
z^{2 m} F\left(z^{-1}\right) & =z^{2 m}\left(P\left(z^{-1}\right)^{2}-z^{-m+n} Q\left(z^{-1}\right)^{2}\right) \\
& =z^{2 m}\left(z^{-2 m} P(z)^{2}-z^{-m+n} z^{-2 n} Q(z)^{2}\right) \\
& =P(z)^{2}-z^{m-n} Q(z)^{2}=F(z) .
\end{aligned}
$$

Suppose that $\left|P\left(z_{0}\right)\right|^{2}=\left|Q\left(z_{0}\right)\right|^{2}$ for some $z_{0}$ with $\left|z_{0}\right|=1$ and $z_{0} \neq 1$. Using $\overline{z_{0}}=1 / z_{0}$ and $P(z), Q(z)$ self-reciprocal, we have

$$
\begin{aligned}
0 & =P\left(z_{0}\right) \overline{P\left(z_{0}\right)}-Q\left(z_{0}\right) \overline{Q\left(z_{0}\right)}=P\left(z_{0}\right) P\left(z_{0}^{-1}\right)-Q\left(z_{0}\right) Q\left(z_{0}^{-1}\right) \\
& =z_{0}^{-m} P\left(z_{0}\right)^{2}-z_{0}^{-n} Q\left(z_{0}\right)^{2}=z_{0}^{-m}\left(P\left(z_{0}\right)^{2}-z_{0}^{m-n} Q\left(z_{0}\right)^{2}\right) \\
& =z_{0}^{-m} F\left(z_{0}\right),
\end{aligned}
$$

which completes the proof.

Example 3. Let $P(z)=z^{4}+1$ and $Q(z)=z^{2}+1$. For $z_{0}=\frac{1 \pm i \sqrt{3}}{2}$ and $z_{1}=\frac{-1 \pm i \sqrt{3}}{2}$, we may compute that

$$
\left|P\left(z_{0}\right)\right|=\left|Q\left(z_{0}\right)\right|=\left|P\left(z_{1}\right)\right|=\left|Q\left(z_{1}\right)\right|=1 .
$$

Also the minimal polynomials of $z_{0}$ and $z_{1}$ are

$$
z^{2}-z+1
$$

and

$$
z^{2}+z+1
$$

respectively. Now we can confirm that the two polynomials above, $z^{2} \pm z+1$, are factors of

$$
\left(z^{4}+1\right)^{2}-z^{2}\left(z^{2}+1\right)^{2}=(z-1)^{2}(z+1)^{2}\left(z^{2}+z+1\right)^{2}\left(z^{2}-z+1\right)^{2} .
$$

Example 4. Consider the self-reciprocal polynomials

$$
z^{3}+1 \text { and } z^{2}+z+1
$$


having all their zeros on $U$. By Theorem 1, a complex number $z_{0}$ on $U$ with $\left|z_{0}^{3}+1\right|=\left|z_{0}^{2}+z_{0}+1\right|$ must have the minimal polynomial

$$
F(z)=z^{6}-z^{5}-2 z^{4}-z^{3}-2 z^{2}-z+1
$$

since

$$
\left(z^{3}+1\right)^{2}-z\left(z^{2}+z+1\right)=F(z)
$$

and $F(z)$ is irreducible.

The minimal polynomials of $z_{0}$ and $z_{1}$ in Example 3 have all their zeros on $U$. However we may verify that $F(z)$ in Example 4 has two zeros not on $U$. Hence it is natural to ask which self-reciprocal polynomials $P(z)$ and $Q(z)$ in Theorem 1 give the minimal polynomial of $z_{0}$ having all its zeros on $U$. We now provide two examples of such pairs of polynomials:

(1) $P(z)=z^{n+k}+1, Q(z)=z^{n}+1$.

For $k \geq 1$,

$$
\begin{aligned}
& \left(z^{n+k}+1\right)^{2}-z^{k}\left(z^{n}+1\right)^{2} \\
= & \left(z^{k}-1\right)\left(z^{2 n+k}-1\right) \\
= & (z-1)^{2}\left(z^{k-1}+z^{k-2}+\cdots+1\right)\left(z^{2 n+k-1}+z^{2 n+k-2}+\cdots+1\right) .
\end{aligned}
$$

(2) $P(z)=\frac{z^{m}-1}{z-1}, Q(z)=\frac{z^{n}-1}{z-1}$.

For $m \geq n$,

$$
\begin{aligned}
& \left(\frac{z^{m}-1}{z-1}\right)^{2}-z^{m-n}\left(\frac{z^{n}-1}{z-1}\right)^{2} \\
= & \frac{\left(z^{m-n}-1\right)\left(z^{m+n}-1\right)}{(z-1)^{2}} \\
= & \left(z^{m-n-1}+z^{m-n-2}+\cdots+1\right)\left(z^{m+n-1}+z^{m+n-2}+\cdots+1\right) .
\end{aligned}
$$

For the proof of Theorem 2, we need the following lemma which is the Nikolskii-type inequality (see Theorem 2.6 of [1]) for the class of real trigonometric polynomials of degree at most $n$.

Let $\mathbf{K}:=\mathbb{R}(\bmod 2 \pi)$. For $f \in C(\mathbf{K})$, let

$$
\|f\|_{p}:=\left(\int_{0}^{2 \pi}|f(\theta)|^{p} d \theta\right)^{1 / p}, \quad 0<p<\infty .
$$

Lemma 5. Let $T_{n}$ be a real trigonometric polynomial of degree at most $n$, and $0<q \leq p \leq \infty$. Then we have

$$
\left\|T_{n}\right\|_{p} \leq\left(\frac{2 r n+1}{2 \pi}\right)^{\frac{1}{q}-\frac{1}{p}}\left\|T_{n}\right\|_{q},
$$


where $r:=r(q)$ is the smallest integer not less than $q / 2$.

Proof of Theorem 2. For even integers $m$ and $n$, let

$$
P(z)=\sum_{k=0}^{m} a_{k} z^{k}, \quad Q(z)=\sum_{k=0}^{n} b_{k} z^{k}
$$

be integral self-reciprocal polynomials with $\operatorname{deg} P(z)=m \geq n=\operatorname{deg} Q(z)$. Suppose that

$$
|P(z)| \neq|Q(z)|
$$

for all $z \in \mathbb{C}$ with $|z|=1$. Write $F(z)=F_{1}(z) F_{2}(z)$, where

$$
F_{1}(z)=P(z)-z^{\frac{m-n}{2}} Q(z), \quad F_{2}(z)=P(z)+z^{\frac{m-n}{2}} Q(z) .
$$

Then both $F_{1}(z)$ and $F_{2}(z)$ have no zeros on $U$ and $\operatorname{deg} F_{1}(z)=\operatorname{deg} F_{2}(z)=m$. Now we have

Since, for $z=e^{i \theta}$, we have

$$
\frac{F_{1}(z)}{z^{\frac{m}{2}}}=\frac{P(z)}{z^{\frac{m}{2}}}-\frac{Q(z)}{z^{\frac{n}{2}}} .
$$

$$
\begin{aligned}
\frac{P(z)}{z^{\frac{m}{2}}} & =a_{\frac{m}{2}}+a_{\frac{m}{2}-1}\left(z+\frac{1}{z}\right)+a_{\frac{m}{2}-2}\left(z^{2}+\frac{1}{z^{2}}\right)+\cdots+a_{0}\left(z^{\frac{m}{2}}+\frac{1}{z^{\frac{m}{2}}}\right) \\
& =a_{\frac{m}{2}}+2\left(a_{\frac{m}{2}-1} \operatorname{Re} z+\cdots+a_{0} \operatorname{Re} z^{\frac{m}{2}}\right) \\
& =a_{\frac{m}{2}}+2\left(a_{\frac{m}{2}-1} \cos (\theta)+\cdots+a_{0} \cos \left(\frac{m}{2} \theta\right)\right)
\end{aligned}
$$

and similarly

$$
\frac{Q(z)}{z^{\frac{n}{2}}}=b_{\frac{n}{2}}+2\left(b_{\frac{n}{2}-1} \cos (\theta)+\cdots+b_{0} \cos \left(\frac{n}{2} \theta\right)\right) .
$$

Since $F_{1}(z)$ has no zeros on $U$,

$$
\begin{aligned}
T(\theta):= & \frac{F_{1}(z)}{z^{\frac{m}{2}}}=\frac{P(z)}{z^{\frac{m}{2}}}-\frac{Q(z)}{z^{\frac{n}{2}}} \\
= & \left(a_{\frac{m}{2}}+2\left(a_{\frac{m}{2}-1} \cos (\theta)+\cdots+a_{0} \cos \left(\frac{m}{2} \theta\right)\right)\right) \\
& -\left(b_{\frac{n}{2}}+2\left(b_{\frac{n}{2}-1} \cos (\theta)+\cdots+b_{0} \cos \left(\frac{n}{2} \theta\right)\right)\right) \\
= & a_{\frac{m}{2}}-b_{\frac{n}{2}}+2 \sum_{k=1}^{\frac{n}{2}}\left(a_{\frac{m}{2}-k}-b_{\frac{n}{2}-k}\right) \cos (k \theta) \\
& +2 \sum_{k=\frac{n}{2}+1}^{\frac{m}{2}} a_{\frac{m}{2}-k} \cos (k \theta)
\end{aligned}
$$

has no any real zeros. Without loss of generality we may assume that $T$ is positive on the real line. Then we have

$$
\|T\|_{1}=\int_{0}^{2 \pi} T(\theta) d \theta=2 \pi\left(a_{\frac{m}{2}}-b_{\frac{n}{2}}\right) .
$$


Using the Parseval formula, we also have

$$
\begin{aligned}
\|T\|_{2}^{2}= & \int_{0}^{2 \pi} T(\theta)^{2} d \theta=\frac{\pi}{2}\left(a_{\frac{m}{2}}-b_{\frac{n}{2}}\right)^{2} \\
& +4 \pi\left[\sum_{k=1}^{\frac{n}{2}}\left(a_{\frac{m}{2}-k}-b_{\frac{n}{2}-k}\right)^{2}+\sum_{k=\frac{n}{2}+1}^{\frac{m}{2}} a_{\frac{m}{2}-k}^{2}\right] .
\end{aligned}
$$

By Lemma 5,

$$
\|T\|_{2}^{2} \leq\left(\frac{m+1}{2 \pi}\right)\|T\|_{1}^{2}
$$

and so

$$
\begin{aligned}
& \frac{1}{2}\left(a_{\frac{m}{2}}-b_{\frac{n}{2}}\right)^{2}+4\left[\sum_{k=1}^{\frac{n}{2}}\left(a_{\frac{m}{2}-k}-b_{\frac{n}{2}-k}\right)^{2}+\sum_{k=\frac{n}{2}+1}^{\frac{m}{2}} a_{\frac{m}{2}-k}^{2}\right] \\
\leq & \left(\frac{m+1}{2 \pi}\right) 4 \pi\left(a_{\frac{m}{2}}-b_{\frac{n}{2}}\right)^{2}=2(m+1)\left(a_{\frac{m}{2}}-b_{\frac{n}{2}}\right)^{2},
\end{aligned}
$$

i.e.,

$$
\left(a_{\frac{m}{2}}-b_{\frac{n}{2}}\right)^{2} \geq \frac{8}{4 m+3}\left[\sum_{k=1}^{\frac{n}{2}}\left(a_{\frac{m}{2}-k}-b_{\frac{n}{2}-k}\right)^{2}+\sum_{k=\frac{n}{2}+1}^{\frac{m}{2}} a_{\frac{m}{2}-k}^{2}\right] .
$$

Using $F_{2}(z)$ having no zeros on $U$, we follow above method to get

$$
\left(a_{\frac{m}{2}}+b_{\frac{n}{2}}\right)^{2} \geq \frac{8}{4 m+3}\left[\sum_{k=1}^{\frac{n}{2}}\left(a_{\frac{m}{2}-k}+b_{\frac{n}{2}-k}\right)^{2}+\sum_{k=\frac{n}{2}+1}^{\frac{m}{2}} a_{\frac{m}{2}-k}^{2}\right],
$$

which completes the proof.

\section{References}

[1] R. A. DeVore and G. G. Lorentz, Constructive Approximation, Springer-Verlag, Berlin, 1993

[2] S.-H. Kim, The zeros of certain family of self-reciprocal polynomials, Bull. Korean Math. Soc. 44 (2007), no. 3, 461-473.

Department of Mathematics

SOOKMYUNG WOMEN'S UNIVERSITY

SEOUl 140-742, Korea

E-mail address: shkim17@sookmyung.ac.kr 